\title{
Correlating brain metabolism with stereotypic and locomotor behavior
}

\author{
SUSAN M. MELNICK and DIANA L. DOW-EDWARDS \\ State University of New York Downstate Medical Center, Brooklyn, New York
}

\begin{abstract}
Many studies have shown that developmental cocaine exposure alters brain function and behavior; the present study examined the relationship between brain metabolism and behavioral responses to drug challenge. SKF 82958, a selective D1 dopamine agonist, was administered to preweaning cocaineexposed (50 mg/kg/day) rats and controls at 60 days of age. Deoxyglucose was administered $30 \mathrm{~min}$ later, during the peak behavioral response, to measure brain functional activity. Pearson product-moment correlations of behavior (locomotor activity and stereotypic behavior) with rates of glucose metabolism in components of the nigrostriatal and mesolimbic circuits were analyzed. The analysis revealed that under saline-challenge conditions in control animals, rates of metabolism in mesolimbic regions are positively correlated to rates of locomotor activity, whereas in cocaine-treated rats, these correlations were absent. Following SKF challenge, a different pattern was seen; locomotor activity or stereotypic behavior was not correlated with mesolimbic or nigrostriatalmetabolism, respectively, in controls but was positively correlated in cocaine-treated rats. Therefore, cocaine exposure during development enhances the coupling of metabolism in components of the mesolimbic and nigrostriatal dopamine systems with the behavioral output associated with these systems under drug-challenge conditions. This may be due to loss of inhibitory influences within the mesolimbic and nigrostriatal systems. Thus, the correlation of behavior and cerebral glucose metabolism provides a unique way of examining the effect of developmental cocaine exposure.
\end{abstract}

Although cocaine use has decreased on a nationwide basis, there is still a significant number of infants and children who were exposed during development. Infant outcome measures showing adverse effects of cocaine range from mild and temporary neurobehavioral symptoms to severe growth and mental retardation (Dow-Edwards, Mayes, Spear, \& Hurd, 1999). Several groups have identified consistent neurobehavioral changes in the realm of attention, affect, arousal, and state orientation (Lester, LaGasse, Freier, \& Brunner, 1996; Mayes, Grillon, Granger, $\&$ Schottenfeld, 1998). Neurochemical and functional abnormalities have been identified in animal models utilizing low to moderate doses of cocaine during development. Cocaine exposure during early development has been shown to uncouple the D1 dopamine receptor from its effector protein complex (Wang, Runyan, Yadin, \& Friedman, 1995), and studies have documented a reduction in behavioral responses to D1 dopamine agonists in rats exposed to cocaine during development (e.g., DowEdwards \& Busidan, 2001).

This work was supported by NIDA Grant 10990. Cocaine $\mathrm{HCl}$ was provided by the Research Triangle Institute. The technical efforts of April Jackson, Mariya Kreymerman, and Alexandra Guillaume are gratefully acknowledged. Statistical guidance was provided by Dr. Jeremy Weedon. Correspondence should be addressed to D. L. Dow-Edwards, Department of Physiology/Pharmacology, State University of New York Downstate Medical Center, 450 Clarkson Avenue, Box 29, Brooklyn, NY 11203 (e-mail: diana.dow-edwards@downstate.edu).
The qualitative relationship between behavior and brain metabolism has previously been explored (e.g., Porrino, Lucignani, Dow-Edwards, \& Sokoloff, 1984). The dose-response pattern of amphetamine-induced locomotor activity was similar to the dose-response pattern of brain glucose metabolism in the nucleus accumbens, whereas stereotypy scores related to metabolism in the caudate nucleus. Direct within-subjects quantitative comparisons could not be made since separate groups of animals were analyzed for behavior and brain metabolism.

In order to determine whether the dampened behavioral response in cocaine-treated rats may be related to a dampened cerebral functional response, adult male rats that had been administered cocaine or vehicle during the preweaning period were administered either saline or SKF 82958, a selective dopamine D1 receptor agonist. At the peak behavioral response, animals were evaluated for brain metabolic activity using the deoxyglucose method (Sokoloff et al., 1977); behavior (locomotor activity and stereotypy) was recorded simultaneously.

\footnotetext{
Method

Animals. Male Sprague-Dawley rats were offspring of female dams purchased from Charles River Laboratories (VAF strain; Wilmington, MA). Animals were housed in plastic tubs with wood chips within our AAALAC-approved temperature- $\left(20^{\circ}-22^{\circ} \mathrm{C}\right)$ and humidity-controlled vivarium on a 12-h light:dark cycle (lights on at 0700 h). Purina Rat Chow and water were available ad lib except where noted. All procedures were preapproved by the SUNY Institutional Animal Care and Use Committee. On Postnatal Day 1 (PND1; day of birth), animals were sexed, weighed, and toe-clipped for iden-
} 

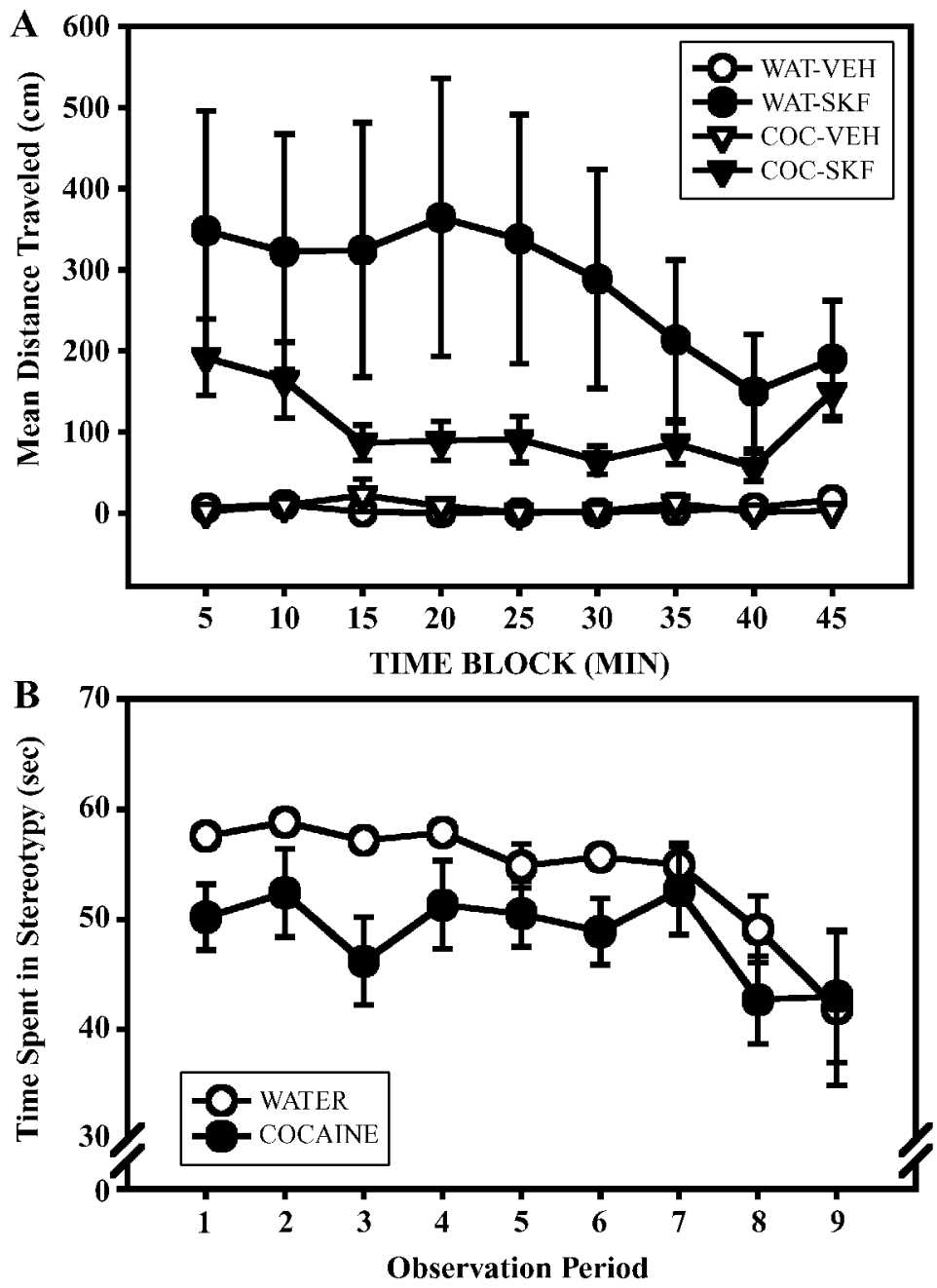

Figure 1. (A) Mean $( \pm S E M)$ total distance traveled $(\mathrm{cm} / 5 \mathrm{~min})$ for each pretreatment and drug group $(n=9-11)$ across the period of 2-DG circulation. Animals injected with saline showed low levels of locomotor activity. Under SKF conditions, water-pretreated animals showed more locomotor activity than did cocaine-pretreated animals. (B) Mean $( \pm S E M)$ time spent (seconds) in low- and medium-intensity stereotyped behavior for each treatment group $(n=11$ each) injected with SKF during each 1-min observation period across the period of 2-DG circulation. Water-pretreated animals spent more time in stereotyped behavior, especially during the first three time points $(15 \mathrm{~min})$, than did the cocaine-pretreated animals.

tification purposes. Litters were culled to 8 ( 4 males:4 females where possible). From PND11 through PND20, all animals within a litter received daily subcutaneous injections of either sterile water (10 ml/kg; Baxter, Deerfield, IL) or $50 \mathrm{mg} / \mathrm{kg}$ cocaine $\mathrm{HCl}$ (NIDA, Research Triangle Park, NC). Litters were weaned on PND21, separated into same-sex pairs, and weighed every 4 days until PND60.

Procedure. Male offspring were implanted with catheters (PE-50 polyethylene tubing) in both the femoral artery and vein under halothane anesthesia on PND60 following an overnight fast (DowEdwards, Freed-Malen, \& Hughes, 1993; Frick \& Dow-Edwards, 1995). Catheters were subdermally inserted to the nape of the neck to allow free access for blood sampling and unrestricted animal movement. Animals were allowed to recover under a heat lamp and given access to water 30 min after surgery. Catheters were flushed regularly with heparin-salin e (100 units/ml; Elkins-Sinn, Inc., Cherry Hill, NJ). After at least $2 \mathrm{~h}$ following surgery, animals were injected intraperitoneally with either $0.9 \%$ physiological saline ( $2 \mathrm{ml} / \mathrm{kg}$; Baxter, Deerfield, IL) or $5.0 \mathrm{mg} / \mathrm{kg} \mathrm{SKF} 82958$ (Sigma Chemical Co., St. Louis, MO). Subjects were then placed within a Versamax activity monitor (VMRXYZ16; Accuscan Instruments, Inc., Columbus, $\mathrm{OH}$ ) and videotaped with a VHS RCA camcorder (CC4352) for $30 \mathrm{~min}$. Vertical sensors were not connected. At 15 min post-drug challenge, animals were measured for arterial blood pressure (MicroMed, Inc., Louisville, KY), arterial blood gases (Radiometer ABL5 blood gas analyzer; Radiometer America, Inc., Westlake, OH), baseline glucose level (Beckman Glucose 


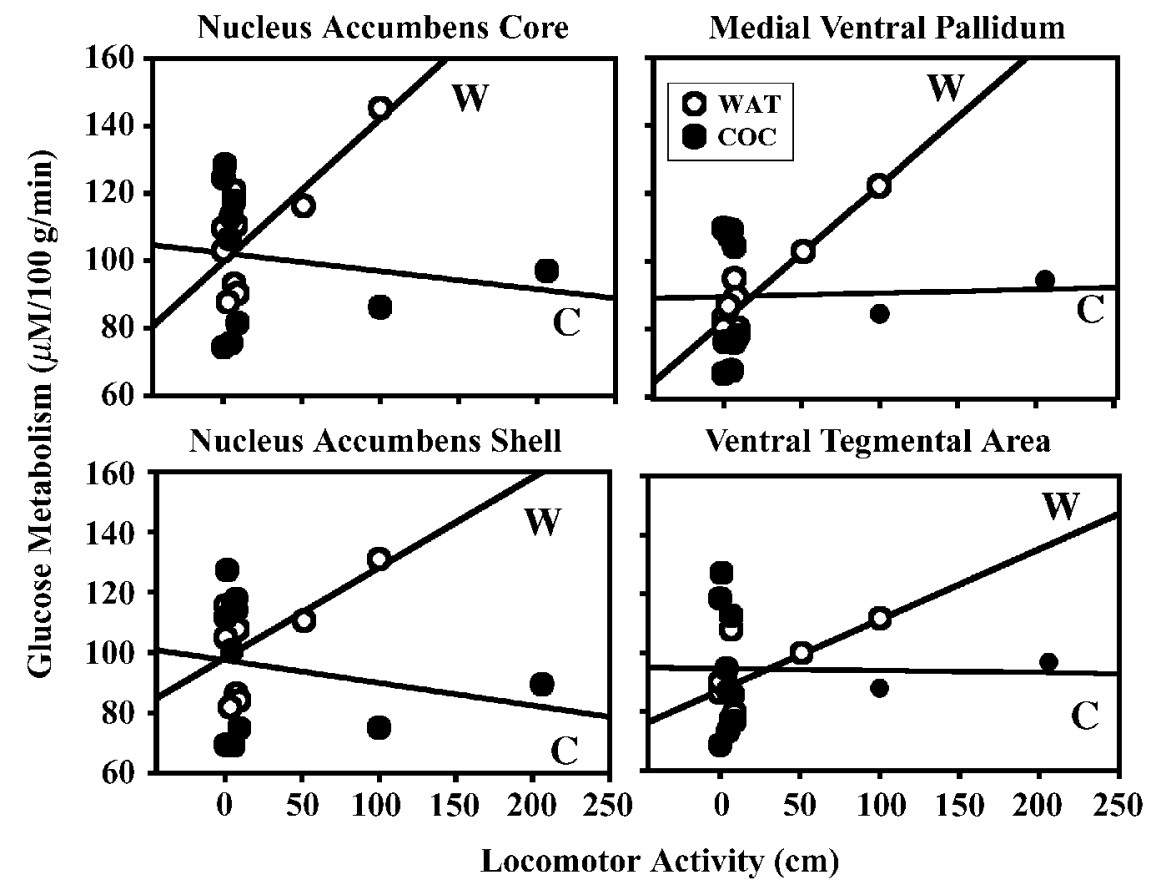

Figure 2. Scatterplots of locomotor activity versus glucose metabolism under saline-injected conditions in individual water- and cocaine-pretreated animals are shown. Featured areas are the nucleus accumbens core and shell at $1.6 \mathrm{~mm}$ rostral to Bregma, the medial ventral pallidum, and the ventral tegmental area. Regression lines are shown for each pretreatment group. The water controls $(n=9)$ showed a positive relationship between locomotor activity and metabolism; the cocaine-pretreated animals $(n=10)$ showed a flat relationship. There were statistically significant differences in the correlations of the water and cocaine groups in the nucleus accumbens core and the medial ventral pallidum $(p<.05)$.

Analyzer 2; Beckman Instruments, Inc., Brea, CA), and finally rectal body temperature (YSI 73ATA; Yellow Springs Instruments, Yellow Springs, CO).

Following the 30-min baseline period, each animal was injected with $125 \mu \mathrm{Ci} / \mathrm{kg}$ body weight $\left[{ }^{14} \mathrm{C}\right] 2$-deoxyglucose (2-DG; specific activity $55 \mathrm{mCi} / \mathrm{mM}$, New England Nuclear, Boston, MA) through the venous catheter and two timers were started. This administration did not disrupt ongoing behaviors. Arterial blood samples were collected for determination of plasma ${ }^{14} \mathrm{C}$ using the Beckman LS1800 scintillation counter and plasma glucose (Glucose Analyzer 2; Beckman Instruments, Inc., Brea, CA). Animals were freely moving during the period of 2-DG circulation. After the 45-min sample, animals were sacrificed with a lethal dose of sodium pentobarbital IV $(60 \mathrm{mg})$. The brains were immediately removed and frozen in 2-methylbutane at $-20^{\circ} \mathrm{C}$ for $5 \mathrm{~min}$. Brains were coated with matrix and stored at $-70^{\circ} \mathrm{C}$ until they were sectioned coronally at $20 \mu \mathrm{m}$ for deoxyglucose autoradiography and histology. Sections mounted on coverglass were exposed to Kodak MinR film along with calibrated ${ }^{14} \mathrm{C}$ standards (Amersham, $\mathrm{Ar}-$ lington Heights, IL) for 12 days. After film development, brain areas of interest were analyzed for glucose metabolism using the Imaging Research MCID M5 system (St. Catharines, ON, Canada) by S.M.M., who was blind as to the experimental treatment of the animals. Structures were based upon the atlas of Paxinos and Watson (1986) and alternate thionin-stained sections. At least four sections per region per animal were measured.

Behavioral measures. Videotapes of the behavioral sessions were blindly observed (by S.M.M.) using Noldus Observer 3.0 software (Wageningen, The Netherlands) to collect data on time spent in low- and medium-intensity stereotyped behavior in nine 1-min observation periods approximately every 5 min during the period of 2-DG circulation. Low-intensity stereotyped behavior was defined as a slight waving of the head beyond sniffing ( $\geqslant$ $2 \mathrm{sec}$ ); medium-intensity was defined as constant head weaving and slow, patterned repetitive movement ( $\geqslant 2 \mathrm{sec})$. Intraobserver reliability was determined to be $r=.98$ from the rescoring of six random sessions. Total distance traveled in centimeters was collected by the Accuscan activity monitors in 5-min periods.

Statistical analyses. Locomotor activity data were analyzed in a mixed measures analysis of variance (ANOVA); the levels were pretreatment (water or cocaine), drug (saline or SKF), and time (nine 5-min time blocks). Stereotyped behavior was analyzed in a pretreatment (water or cocaine) $\times$ time (nine 1-min observation periods) ANOVA since no stereotypy was observed in saline-injected rats. Within each treatment and drug group, Pearson product-moment correlations and associated Bonferroni-corrected $p$ values were calculated for glucose metabolism in components of the mesolimbic and nigrostriatal circuits and behavior (stereotypy and locomotor activity) using SYSTAT. Differences in correlations between pretreatment groups were analyzed using the formula developed by R. A. Fisher (Howell, 1987). Values of $p=.05$ were considered statistically significant.

\section{Results}

Overall, in mediodorsal thalamus, medial ventral pallidum, and ventral tegmental area, SKF increased metabolism (data not shown). In other regions, such as within 


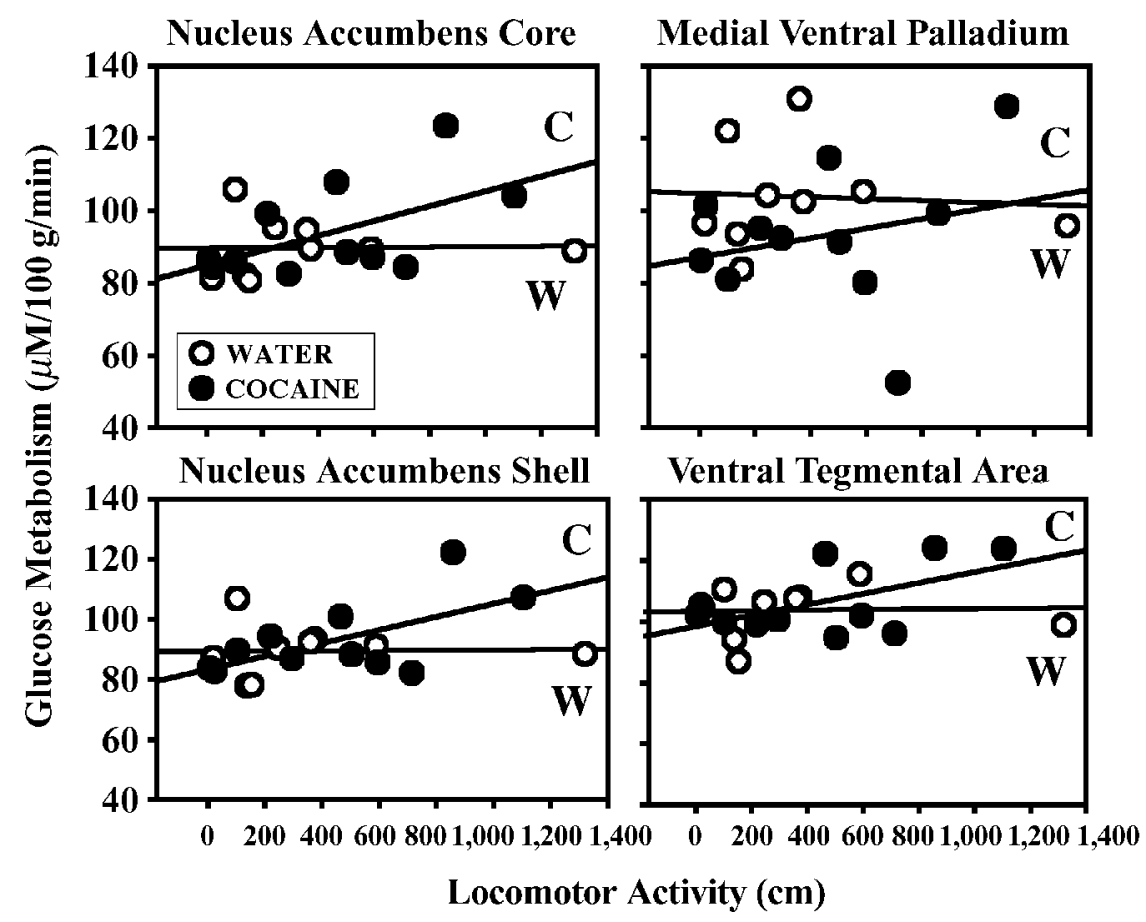

Figure 3. Scatterplots of locomotor activity versus glucose metabolism in mesolimbic areas for water- and cocaine-pretreated animals following SKF injections. Cocaine-pretreated animals $(n=11)$ showed an overall positive relationship between locomotor activity and behavior; water-pretreated animals $(n=9)$ showed a flat relationship. Featured areas as in Figure 2.

the nigrostriatal circuit, SKF had no effect on metabolism in dopamine-rich terminal zones (e.g., the caudateputamen), whereas metabolism in substantia nigra, globus pallidus, and so on, was increased (Melnick \& DowEdwards, 2003). In general, cocaine pretreatment only dampened the metabolic response to SKF seen in rostral areas of the mesolimbic system of the controls (Melnick \& Dow-Edwards, 2003).

Figure 1 shows the locomotor and stereotypic behaviors during the 45-min period of 2-DG circulation. In general, cocaine dampened the behavioral responses to SKF. The ANOVA revealed a significant interaction of pretreatment, drug, and time period $[F(8,296)=2.288, p=.025]$; saline-injected (both control and cocaine-pretreated) animals showed very little locomotor activity, but under SKF conditions the control animals showed more locomotor activity than did the cocaine-pretreated animals (Panel A). Stereotyped behavior (Panel B) occurred only following SKF injections, and control animals showed more stereotypic behavior than did cocaine-pretreated animals, especially during the first $15 \mathrm{~min}$ of 2 -DG circulation $[F(1,20)=5.553, p=.029]$.

Figures 2-4 are scatterplots of individual animals' behavior (collected during the first $15 \mathrm{~min}$ post-2-DG administration) and the rates of metabolism in mesolimbic (for locomotor activity) and nigrostriatal (for stereotypy) systems. The best-fit regression lines for each treatment group are shown. Following saline injections, controls showed a positive relation between locomotor activity and metabolism in several mesolimbic regions, including the nucleus accumbens core and shell (at $1.6 \mathrm{~mm}$ rostral to Bregma), the medial ventral pallidum, and the ventral tegmental area; the cocaine-exposed rats generally showed a flat relationship (Figure 2). Significantly higher correlations were seen in the water-pretreated controls than in the cocaine-pretreated animals between locomotor activity and metabolism in the medial ventral pallidum and the nucleus accumbens core $(p<.05)$.

Following SKF injections, controls generally showed a flat relationship between metabolism and locomotor activity and stereotypy; the cocaine-exposed rats showed slight positive relationships for both the mesolimbic and nigrostriatal circuits (Figures 3 and 4). However, none of these correlations were statistically significant. In addition, there were no significant differences between the pretreatment groups in the correlations of locomotor activity and brain metabolism under SKF conditions (Figure 3). A linear regression analysis revealed that across all the regions of the mesolimbic pathway, the cocaineexposed animals showed a reliably steeper slope than did the controls $[F(1,13.7)=5.44, p=.036]$, suggesting that as locomotor behavior increased, the cocaine-exposed animals showed higher rates of glucose metabolism in areas known to mediate this behavior. However, within individual brain regions, there were no significant differences between water controls and cocaine-pretreated rats. 


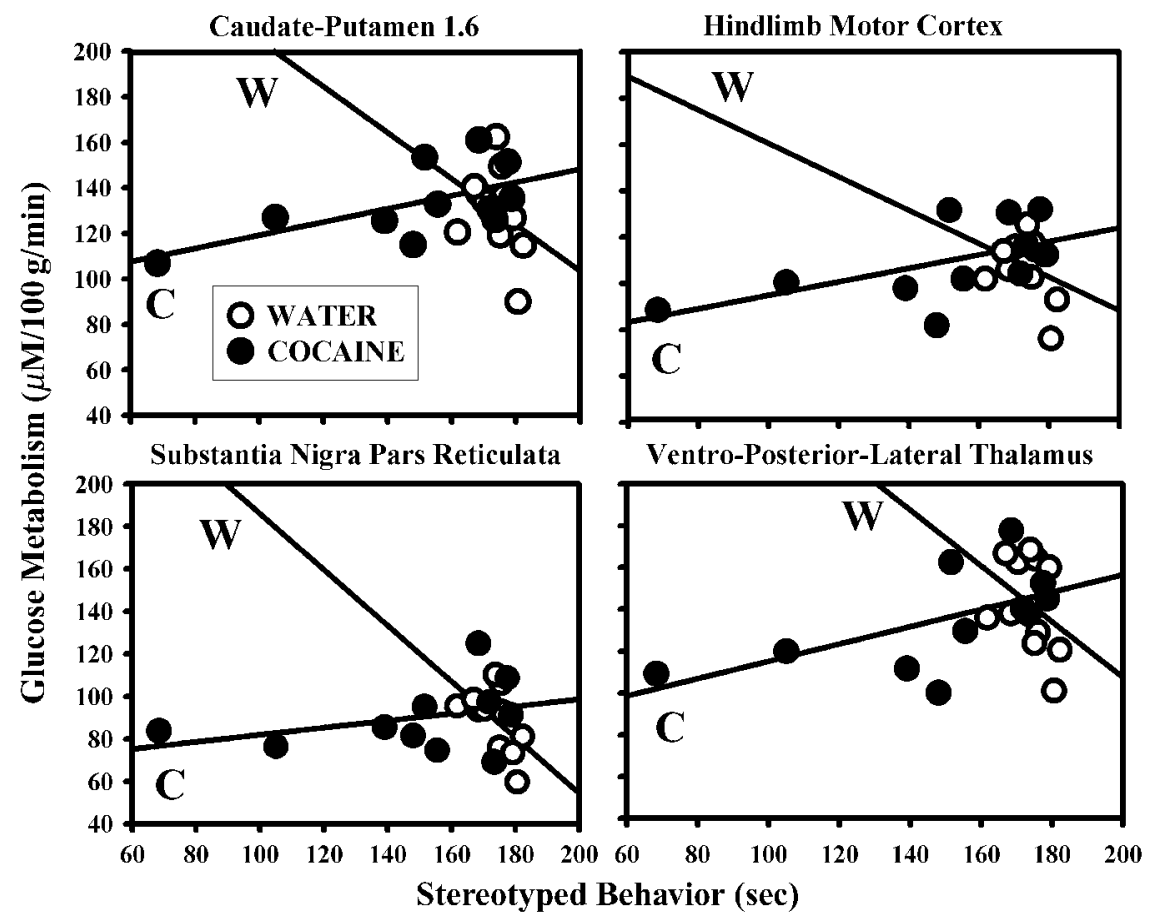

Figure 4. Scatterplots (stereotyped behavior vs. metabolism) for the pretreated groups administered SKF. Regions of the nigrostriatal system, including the caudate-putamen at $1.6 \mathrm{~mm}$ from bregma, hindlimb motor cortex, substantia nigra pars reticulata, and ventroposterior lateral nucleus of the thalamus are shown. Cocaine-pretreated animals $(n=11)$ showed a positive relationship between stereotypy and metabolism; water-pretreated animals $(n=11)$ showed a flat relationship. These differences were statistically significant $(p<.05)$.

An examination of stereotypic behavior (Figure 4) showed that cocaine-pretreated animals had significantly higher correlations than did the water controls of stereotypic behavior and brain metabolism in areas of the caudate-putamen $1.6 \mathrm{~mm}$ rostral to Bregma, hindlimb motor cortex, substantia nigra pars reticulata, centromedial nucleus of the thalamus, and ventroposterior lateral nucleus of the thalamus $(p<.05)$. Although the correlations themselves were not significant, there were significant differences between the cocaine-pretreated group and the controls. Thus, the cocaine-pretreated animals showing increased stereotypic behavior also showed relatively higher glucose metabolic rates in all nigrostriatal regions; this relationship tended to be negative in the controls.

\section{Discussion}

In controls under saline-challenge conditions, the coupling of metabolism and locomotor activity was weakly positive for components of the mesolimbic system (Figure 2). Following SKF administration, a flat relationship between metabolism and locomotor activity or stereotypy was seen in controls, perhaps due to glucose use by both excitatory and inhibitory processes (Figures 3 and 4). Cocaine exposure during development appears to strengthen the coupling of metabolism with the behav- ioral output, especially after SKF administration. This may be due to loss of inhibitory influences in both mesolimbic and nigrostriatal circuits.

Similar to previous reports (e.g., Dow-Edwards \& Busidan, 2001), the present study supported a dampened behavioral response (locomotor activity and stereotyped behavior) to selective D1 receptor agonists following preweaning cocaine exposure (Figure 1).

The observation of both behavior and brain glucose metabolism in the same animals allows for a quantitative comparison as opposed to merely qualitative comparisons. Brain metabolism did not always mirror the pattern of the behavioral responses, as suggested previously with amphetamine (Porrino et al., 1984). This difference may be due to the dissimilar mechanisms of the action of amphetamine, an indirect dopamine agonist, and SKF, a selective direct D1 agonist. The present study, using within-animal correlational analyses, showed that cocaine exposure greatly altered the relationship between brain metabolism and behavior-a result that would have been overlooked in the traditional region-by-region (or behavioral) analysis.

The present study showed that preweaning cocaine exposure consistently altered the relationship of behavior and brain metabolism as measured in adult rats and that differences were even more apparent when the nigrostriatal 
and mesolimbic systems were activated by SKF challenge. Modulatory influences such as by serotonin in these pathways have been shown to be disrupted following preweaning cocaine administration (Dow-Edwards, 1998). Thus, correlational analyses of multiple variables in the same individual animals can be a powerful tool to examine the relationship between brain and behavior.

\section{REFERENCES}

Dow-EDWARDS, D. L. (1998). Preweaning cocaine administration alters the adult response to quipazine: Comparison with fluoxetine. Neurotoxicology \& Teratology, 20, 133-142.

Dow-EDwARDs, D. L., \& BusidAN, Y. (2001). Behavioral responses to dopamine agonists in adult rats exposed to cocaine during the preweaning period. Pharmacology, Biochemistry \& Behavior, 70, 23 30.

Dow-Edwards, D. L., Freed-Malen, L. A., \& Hughes, H. E. (1993). Long-term alterations in brain function following cocaine administration during the preweanling period. Developmental Brain Research, 72, 309-313.

Dow-Edwards, D. L., Mayes, L., Spear, L., \& Hurd, Y. (1999). Cocaine and development: Clinical, behavioral and neurobiological perspectives-A symposium report. Neurotoxicology \& Teratology, 21, 481-490.

FrICK, G. S., \& Dow-EDWARDS, D. L. (1995). The effects of cocaine on cerebral metabolic function in periweanling rats: The roles of serotonergic and dopaminergic uptake blockade. Developmental Brain Research, 88, 158-170.
Howell, D. C. (1987). Statistical methods for psychology (2nd ed.). Boston: Duxbury.

Lester, B. M., LaGasse, L., Freier, K., \& Brunner, S. (1996). Studies of cocaine-exposed human infants. NIDA Research Monograph, 164, 175-210.

Mayes, L. C., Grillon, C., Granger, R., \& Schottenfeld, R. (1998). Regulation of arousal and attention in preschool children exposed to cocaine prenatally. Annals of the New York Academy of Sciences, 846, 126-143.

Melnick, S. M., \& Dow-Edwards, D. L. (2003). Blunted metabolic response to SKF 82958 in the mesolimbic system following preweaning cocaine treatment. Developmental Brain Research, 143, 253-259.

PAXINOS, G., \& WATSON, C. (1986). The rat brain in stereotaxic coordinates. New York: Academic Press.

Porrino, L. J., Lucignani, G., Dow-Edwards, D. [L.], \& Sokoloff, L. (1984). Correlation of dose-dependent effects of acute amphetamine administration on behavior and local cerebral metabolism in rats. Brain Research, 307, 311-320.

Sokoloff, L., Reivich, M., Kennedy, C., Des Rosiers, M. H., Patlak, C. S., Pettigrew, K. D., Sakurada, O., \& Shinohara, M. (1977). The $\left[{ }^{14} \mathrm{C}\right] d e o x y g l u c o s e$ method for the measurement of local cerebral glucose utilization: Theory, procedure, and normal values in the conscious and anesthetized albino rat. Journal of Neurochemistry, 28, 897-916.

Wang, H.-Y., Runyan, S., Yadin, E., \& Friedman, E. (1995). Prenatal exposure to cocaine selectively reduces D1 dopamine receptormediated activation of striatal Gs proteins. Journal of Pharmacology \& Experimental Therapeutics, 273, 492-498.

Manuscript received October 31, 2002; revision accepted for publication May 4, 2003.) 